\title{
Integrated Computational Modeling of Water Side Corrosion in Zirconium Metal Clad Under Nominal LWR Operating Conditions
}

\author{
ASGHAR ARYANFAR, ${ }^{1}$ JOHN THOMAS, ${ }^{2}$ ANTON VAN DER VEN ${ }^{2}$ \\ DONGHUA XU, ${ }^{3}$ MOSTAFA YOUSSEF,${ }^{4}$ JING YANG, ${ }^{4}$ BILGE YILDIZ, \\ and JAIME MARIAN ${ }^{1,5}$

\begin{abstract}
1.-University of California, Los Angeles, Los Angeles, CA, USA. 2.-University of California, Santa Barbara, Santa Barbara, CA, USA. 3.-University of Tennessee-Knoxville, Knoxville, TN, USA. 4.-Massachussetts Institute of Technology, Cambridge, MA, USA. 5.-e-mail:
\end{abstract} \\ jmarian@ucla.edu
}

\begin{abstract}
A mesoscopic chemical reaction kinetics model to predict the formation of zirconium oxide and hydride accumulation light-water reactor (LWR) fuel clad is presented. The model is designed to include thermodynamic information from ab initio electronic structure methods as well as parametric information in terms of diffusion coefficients, thermal conductivities and reaction constants. In contrast to approaches where the experimentally observed time exponents are captured by the models by design, our approach is designed to be predictive and to provide an improved understanding of the corrosion process. We calculate the time evolution of the oxide/metal interface and evaluate the order of the chemical reactions that are conducive to a $t^{1 / 3}$ dependence. We also show calculations of hydrogen cluster accumulation as a function of temperature and depth using spatially dependent cluster dynamics. Strategies to further cohesively integrate the different elements of the model are provided.
\end{abstract}

\section{INTRODUCTION}

Oxidation and hydriding performance of zirconium fuel components in light-water reactors (LWRs) may limit the maximum fuel discharge burn-up, which makes corrosion a critical aspect of $\mathrm{Zr}$ materials response in nuclear environments. Although out-of-pile autoclave corrosion tests developed over the years can predict some aspects of inpile corrosion performance of zirconium alloys, these autoclave tests normally underestimate corrosion rates and do not account for irradiation effects. To help bridge the gap between autoclave measurements and in-reactor $\mathrm{Zr}$ corrosion, several models of zirconium alloy corrosion and hydriding processes have been developed over the years. These models focus only on certain elements of the reported observations of corrosion and hydriding, and are generally formulated 'reactively', i.e. to reproduce observed experimental time evolutions without delving into the underlying causes behind the experimental observations. This lack of predictiveness prevents these models from being used for materials evaluation and design. In this paper, we present a corrosion model that provides a first-principles approach to understand corrosion and hydriding performance of Zircaloys in a general manner, based upon the material microstructure.

\section{Oxide Layer Formation and Growth}

Corrosion occurs by diffusion of oxygen and hydrogen into the $\mathrm{Zr}$ metal and chemical reaction with the host atoms according to: ${ }^{1}$

$$
\mathrm{Zr}(\mathrm{s})+x \mathrm{H}_{2} \mathrm{O}(\mathrm{g}) \rightarrow \mathrm{ZrO}_{x}(\mathrm{~s})+x \mathrm{H}_{2}(\mathrm{~g})
$$

where $x$ is a mass balance factor set by the thermodynamics of the $\mathrm{Zr}-\mathrm{O}$ system. The corrosion kinetics is generally interpreted as diffusion controlled, ${ }^{2}$ with a one-third power law dependency with time ${ }^{2-5}$ At a certain thickness, the oxide scale cracks, ${ }^{6,7}$ which opens up pathways for the water to reach the metallic substrate, leading to a loss of the protective layer and a sudden jump in corrosion rates. Fracture is 
attributed to the contribution of several factors, such as (1) compressive stresses in the oxide due to volumetric expansion during oxidation (Pillar-Bedworth ratio of 1.56$),{ }^{2}$ (2) tensile stresses in the oxide layer due to thermal and irradiation induced expansion on the fuel side, and (3) dynamic embrittlement caused by hydride precipitation. ${ }^{8-12}$ Due to the highly complex and synergistic nature of corrosion kinetics, with coupled oxygen/hydrogen transport, diffusion/ reaction kinetics, temperature effects, and complex oxide microstructures, to name but a few, a multipronged approach that includes modeling as an essential element must be adopted to improve our understanding of the process.

In this paper, we present an integrated model of $\mathrm{Zr}$ oxidation and hydriding kinetics starting from first-principles reaction kinetics, fundamental thermodynamics, and parameterization from electronic structure calculations and experiments. First, we describe the thermodynamic modeling that furnishes phase diagrams and structural details. This is followed by a formulation of the chemical reaction model for oxide layer growth, in which the nonstoichiometric oxidation of $\mathrm{Zr}$ to $\mathrm{ZrO}_{x}$ is computed in real time, considering the simultaneous spatial variation in the effective parameters such as diffusivities, thermal conductivities, and reaction kinetics. Electronic structure calculations of the model parameters are then discussed, and the paper concludes with a rate theory study of hydride accumulation in the $\mathrm{Zr}$ metal layer.

\section{THERMODYNAMICS OF Zr OXIDES AND HYDRIDES}

Clad corrosion is a non-equilibrium, multi-scale process that can be viewed as a sequence of phase transformations of the original hcp Zr-based alloy into its various oxides and hydrides due to the ingress of oxygen and hydrogen. Relevant kinetic processes include oxygen and hydrogen diffusion down chemical potential gradients as well as the migration of interfaces separating the alloy from the various oxide and hydride phases. These thermally activated, non-equilibrium processes can be described at a phenomenological level with kinetic laws based on irreversible thermodynamics and/or Cahn-Hilliard-Allen-type descriptions. 6,13

Predicting the dynamical evolution at a phenomenological level, while very well suited for corrosion processes, nevertheless requires accurate thermodynamic and kinetic information, which is often difficult if not impossible to measure experimentally, especially in isolation. It is here where first-principles electronic structure methods can play a crucial role. However, first-principles electronic structure methods only predict properties at zero temperature, while corrosion occurs at elevated temperature where entropy and thermal activation are important. Statistical mechanics is the key link connecting the $0 \mathrm{~K}$ electronic structure properties of a particular phase to its high-temperature thermodynamic and kinetic properties needed in phenomenological theories. The development of powerful statistical mechanics methods and computational tools to predict free energie ${ }^{14,15}$ and diffusion coefficients ${ }^{16,17}$ in complex alloys and multicomponent ionic and semiconducting compounds is relatively recent, but it has opened the door to understanding the rich thermodynamics of the $\mathrm{Zr}$ $\mathrm{O}-\mathrm{H}$ system, as well as to new interpretations of the complex corrosion kinetics under service.

The phases that form during the corrosion of zirconium and its alloys are especially challenging to describe with first-principles statistical mechanics methods. Zirconium forms a rich variety of oxides and hydrides having a range of crystal structures and compositions. $\mathrm{ZrO}_{2}$, for example, is stable in one of three polymorphs at ambient pressure depending on temperature. Zirconium also forms a series of sub-stoichiometric oxides at very low oxygen partial pressures. In fact, $\alpha$-zirconium, which exists in the hcp crystal structure, is able to dissolve oxygen interstitially up to concentrations of $33 \%$, with the dissolved oxygen exhibiting substantial disorder at high temperature, but forming ordered sub-stoichiometric oxides at low temperature. The hydrides are equally complex. $\mathrm{ZrH}_{2}$, for example, undergoes a cubic to tetragonal martensitic transformation upon cooling from high temperature and can tolerate very high vacancy concentrations over the hydrogen sub-lattice at low hydrogen partial pressures. Metastable hydrides are also observed and are likely stabilized as their formation in the early stages of precipitation is accompanied by less coherency strain energy than the hydrides that are more stable under ambient hydrostatic pressure conditions.

The elevated temperatures at which corrosion occurs requires an explicit treatment of entropic contributions arising from thermal excitations over the many available configurational and vibrational degrees of freedom. The oxygen disorder at high temperature in the $\mathrm{ZrO}_{x}$ sub-oxides, for example, is responsible for configurational entropy. Cubic $\mathrm{ZrH}_{2-x}$ will also have a sizable configurational entropic contribution to the free energy due to the many possible ways of arranging hydrogen vacancies over the interstitial sites of an FCC $\mathrm{Zr}$ sublattice. Even more challenging to predict are contributions from vibrational excitations, which are responsible for the structural transformations among the various polymorphs of $\mathrm{ZrO}_{2}$ and $\mathrm{ZrH}_{2}$.

A powerful method to describe configurational and vibrational excitations in multi-component crystalline solids is with the use of effective Hamiltonians. These are mathematical expressions that can be parameterized with first-principles calculations and that can then be used to predict the energies of arbitrary excitations, often with close to first-principles accuracy. This makes effective Hamiltonians invaluable within Monte Carlo 
simulations where thermodynamic averages are estimated. A cluster expansion effective Hamiltonian, ${ }^{18,19}$ for example, is expressed in terms of polynomials of site occupation variables and enables the rapid calculation of the energy of a multicomponent solid as a function of the degree of order among its various constituents. A harmonic phonon Hamiltonian is a similar expansion, expressed in terms of polynomials of the components of atomic displacement vectors belonging to pair clusters. Phonon Hamiltonians are restricted to small perturbations and are accurate for stiff bonds or at low temperatures where vibrational amplitudes are small. Many important high temperature phases, however, exhibit large anharmonic vibrational excitations requiring Hamiltonians that go beyond the harmonic approximation. In this context, a variety of anharmonic lattice dynamical effective Hamiltonians have been developed that are expressed either as a function of lattice Wannier functions, ${ }^{20,21}$ or as a function of symmetrized collective displacement modes of clusters of sites. ${ }^{22}$ These effective Hamiltonians have been especially invaluable in predicting properties of high-temperature phases that exhibit dynamical instabilities at low temperatures.

Figure 1 shows a Zr-O binary phase diagram calculated from first-principles calculations accounting for configurational and vibrational excitations. The configurational degrees of freedom were captured by combining a first-principles parameterized cluster expansion with Monte Carlo simulations. Vibrational excitations were accounted for within the quasi-harmonic approximation, with vibrational free energies explicitly calculated for the stoichiometric oxides and linearly interpolated for the $\mathrm{ZrO}_{x}$ solid solution between $x=0$ and $1 / 2$. The phase diagram shows the remarkably high oxygen solubility of oxygen in hcp-based Zr. At high temperatures, the oxygen forms a solid solution over the octahedral interstitial sites of hcp Zr. At low temperatures, the dissolved oxygen orders at stoichiometric compositions, including $\mathrm{ZrO}_{1 / 6}, \mathrm{ZrO}_{1 / 3}$ and $\mathrm{ZrO}_{1 / 2}$. The $\mathrm{ZrO}_{1 / 6}$ sub-oxide can be viewed as staged phase in that every other layer of interstitial sites is empty while the oxygen in the filled alternating layers are well ordered, forming a $\sqrt{3} a \times \sqrt{3} a$ supercell. In $\mathrm{ZrO}_{1 / 3}$, every layer fills up, but the in-plane ordering is the same as in the filled layers of $\mathrm{ZrO}_{1 / 6}$. The $\mathrm{ZrO}_{1 / 2}$ suboxide maintains a $\sqrt{3} a \times \sqrt{3} a$ supercell ordering perpendicular to the c-axis, but achieves half-filling over the octahedral sites by having layers with $1 / 3$ filling alternated by layers having $2 / 3$ filling. However, as is clear from the calculated phase diagram, this phase is able to tolerate a large degree of offstoichiometry and disorders through a second-order mechanism. The phase diagram also shows a transition temperature for $\mathrm{ZrO}_{2}$ from its monoclinic form $\alpha$ to its tetragonal form $\beta$. This transition temperature was predicted within the quasi-harmonic approximation.

While the $\mathrm{Zr}-\mathrm{O}$ binary has been studied for decades, it was only recently that a new monoxide phase having $\mathrm{ZrO}$ stoichiometry was discovered. Experiments showed the existence of a $\mathrm{ZrO}$

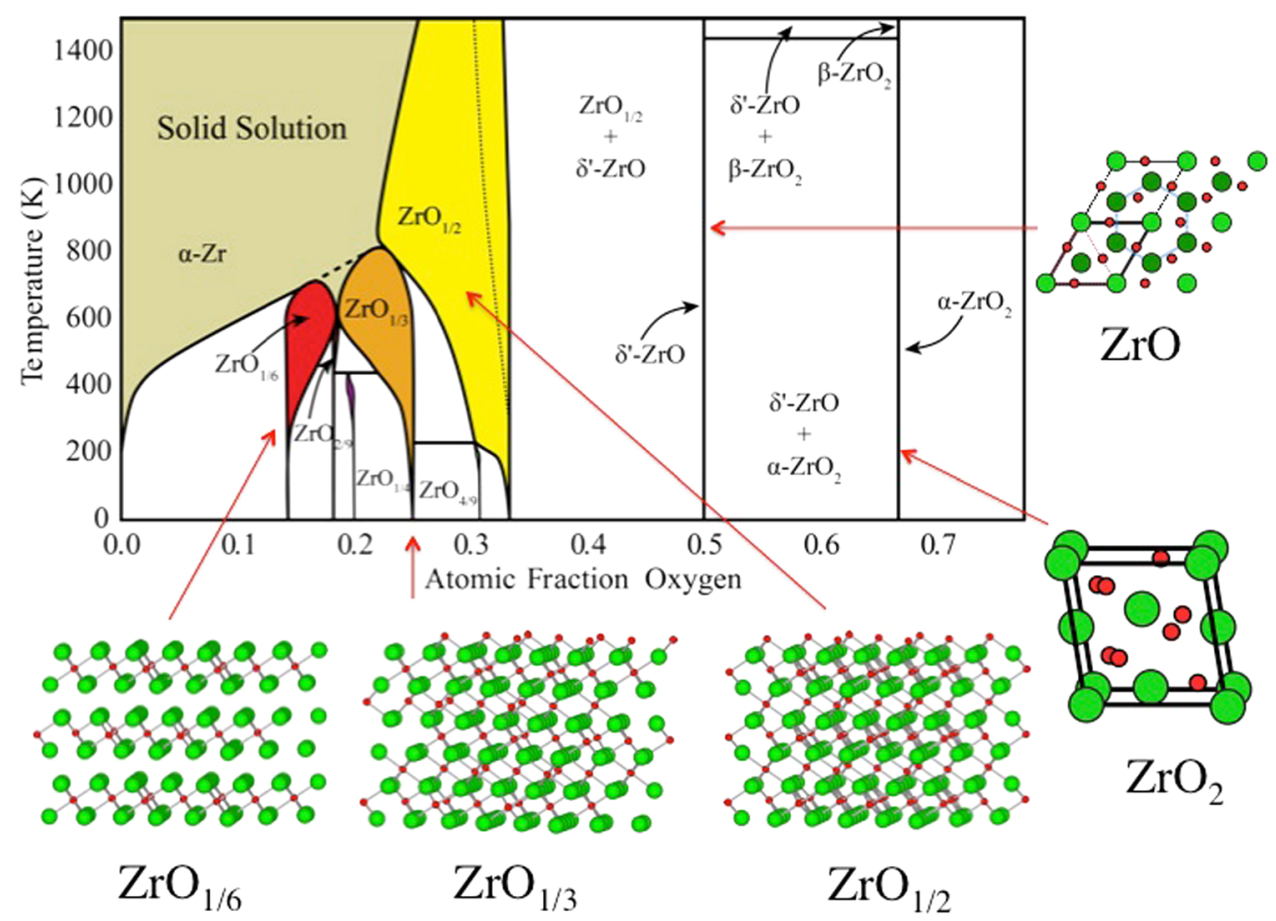

Fig. 1. The calculated Zr-O binary phase diagram. ${ }^{25,26}$ 
monoxide in oxidation microstructures. ${ }^{23,24}$ However, it was with systematic first-principles enumeration and structure search algorithms that the crystal structure of this sub-oxide was unraveled. ${ }^{25}$ This new phase, labeled $\delta$, consists of an omega $\mathrm{Zr}$ sub-lattice (made of alternating honeycomb $\mathrm{Zr}$ layers interleaved with triangular $\mathrm{Zr}$ layers) with the oxygen occupying square pyramidal interstitial sites of the $\mathrm{Zr}$ sub-lattice. The vibrational entropy of this phase was predicted to be lower than that of competing oxides due to the tight packing; however, the phase was found to remain stable until at least about $1500 \mathrm{~K}$. It appears as a line compound in Fig. 1 as it does not tolerate off-stoichiometry through the introduction of oxygen vacancies or interstitials. ${ }^{25}$

The hydrides of $\mathrm{Zr}$ pose even greater challenges to first-principles statistical mechanics, in large part because the important cubic $\mathrm{ZrH}_{2-x}$ form is predicted to be dynamically unstable at low temperatures. In addition to tolerating high vacancy concentrations over the hydrogen sub-lattice, leading to sizable configurational entropic contributions to the free energy, cubic $\mathrm{ZrH}_{2-x}$ also exhibits large anharmonic vibrational excitations that dynamically stabilize its cubic symmetry. Recently, a cluster expansion Hamiltonian describing vibrational degrees of freedom was developed to account for the large anharmonic fluctuations that stabilize high symmetry phases such as cubic $\mathrm{ZrH}_{2} \cdot{ }^{22} \mathrm{Com}$ bined with Monte Carlo simulations, it was possible to predict the experimentally observed tetragonal to cubic phase transformation upon heating. Figure 2a shows the predicted dependence of the unit cell lattice parameters on temperature, clearly showing a second order phase transition from the tetragonal form of $\mathrm{ZrH}_{2}$ to the cubic form. Figure $2 \mathrm{~b}$ shows calculated free energy curves as a function of the $c /$ $a$ ratio. At zero Kelvin, the cubic phase, corresponding to $c / a=1$, is clearly dynamically unstable due to the negative curvature of the free energy. With increasing temperature though, anharmonic vibrational excitations reduce the difference in free energy between the tetragonal phase, corresponding to the minimum at $c / a<1$, and the cubic phase at $c / a=1$. At the second-order transition temperature, the minimum of the free energy shifts to $c /$ $a=1$, rendering the cubic phase thermodynamically stable. It was only upon the introduction of new statistical mechanics tools accounting for anharmonic vibrational excitations that important thermodynamic properties such as the elastic moduli could be calculated for phases such as cubic $\mathrm{ZrH}_{2},{ }^{27}$ which are dynamically unstable at low temperature, but become dynamically stabilized through large vibrational excitations at high temperature.

\section{MESOSCOPIC KINETIC MODEL OF OXIDE LAYER FORMATION AND GROWTH}

The oxygen ions from the electrolysis of the water reach the clad outer surface and diffuse in the $\mathrm{Zr}$ matrix. Free oxygen diffusion occurs preferentially along the grain boundaries, accelerating ion transport with respect to bulk diffusion. As the oxygen concentration builds up, the resulting $\mathrm{Zr}$ and $\mathrm{O}$ mixtures evolve, giving rise first to stable solid solutions, followed by intermetallic structures as well as additional sub-oxide phases such as the (metallic) $\delta$ ' $\mathrm{rO}$ phase. When the atomic fraction of $\mathrm{O}$ approaches the oxide stoichiometric phase boundary in the phase diagram, ${ }^{25}$ the system begins to transform into a substoichiometric form of tetragonal zirconia, $\mathrm{ZrO}_{2-x}$ (with $x<0.02$ ). It is believed that this layer consists of arrays of roughly equiaxed nanocrystals with various stress-minimizing orientations relative to the metallic substrate, stabilized by the relatively large internal surface-to-volume ratio. This metastable phase is soon replaced by a stable monoclinc saturated (stoichiometric) zirconia phase, as more free oxygen is picked up and immobilized into the oxide phase. Multilayer evolution systems are typically treated as interfaceevolving Stefan problems. However, this naturally (a)

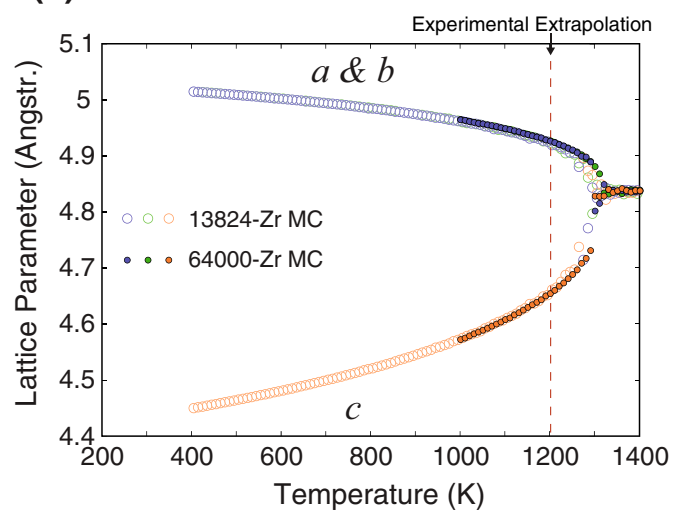

(b)

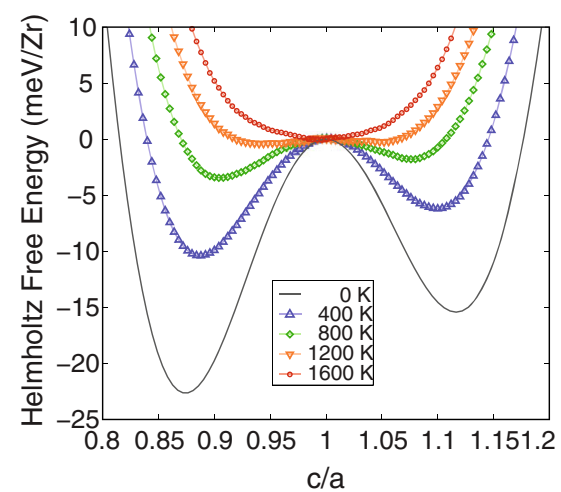

Fig. 2. (a) Calculated lattice parameters as a function of temperature for $\mathrm{ZrH}_{2}$. (b) Calculated free energies of $\mathrm{ZrH} 2$ as a function of $c / a$ ratio at several temperature below and above the second order tetragonal to cubic phase transition. 
leads to a parabolic growth scaling law which is known to not hold for Zircalloy corrosion. Instead, here, we develop a one-dimensional chemical reaction kinetics model.

Throughout the process, the atomic fraction of oxygen (related to the partial pressure) in $\mathrm{Zr}$ is defined as:

$$
X=\frac{[\mathrm{Oi}]}{[\mathrm{Oi}]+[\mathrm{Zr}]}
$$

where [Oi] is the concentration of chemically immobilized oxygen (i.e. not available for diffusion). The value of $X$ for the substoichiometric tetragonal phase denoted by $\mathrm{ZrO}_{2-x}$ that forms first is then given by:

$$
X=\frac{2-x}{3-x}
$$

which corresponds to non-stoichiometric ratio of $X \sim 0.6644$ when $x=0.02$. The value for monoclinic zirconia is a constant at $X=2 / 3$. Therefore, once the oxide layer emerges, oxygen diffusion proceeds on two different media with different physical properties. ${ }^{28}$ This picture including a three-layer system is schematically depicted as a one-dimensional process in Fig. 3.

A temperature gradient exists within the clad, such that the temperature depends on depth, which we denote by the spatial variable $y$. A simple order of magnitude analysis of the values of the thermal diffusivity $\alpha$ and the diffusion coefficient D yields approximately:

$$
\left\{\begin{array}{l}
\alpha=\frac{\kappa}{\rho c_{\mathrm{p}}} \approx 10^{-5} \frac{\mathrm{m}^{2}}{\mathrm{~s}} \\
D \approx 10^{-14} \frac{\mathrm{m}^{2}}{\mathrm{~s}}
\end{array} \rightarrow \frac{\partial T}{\partial t} \gg \frac{\partial[O]}{\partial t}\right.
$$

where $\rho$ is the mass density, $c_{\mathrm{p}}$ is the heat capacity, and $T$ is the absolute temperature. This means that the thermal propagation rate is significantly faster than mass transport of $\mathrm{O}$ in $\mathrm{Zr}$. Hence, we can assume that, at any stage in the zirconium oxide growth, the temperature distribution equilibrates very rapidly on the scale of atom diffusivity. This allows us to discard the time dependence of the thermal profile an any time $(T=T(y))$. With this, the set of parabolic differential equation that expresses the spatio-temporal variation of free oxygen in the system is:

$$
\left\{\begin{array}{l}
\frac{\mathrm{d}[O]}{\mathrm{d} t}=D(T)\left(\frac{\partial^{2}[\mathrm{O}]}{\partial y^{2}}+\frac{Q}{K_{B} T^{2}} \frac{\partial T}{\partial y} \frac{\partial[\mathrm{O}]}{\partial y}\right)-k_{r x n}[\mathrm{O}]^{\beta} \\
\frac{\mathrm{d}[\mathrm{Oi}]}{\mathrm{d} t}=k_{r x n}[O]^{\beta}
\end{array}\right.
$$

where $\beta$ is the reaction order. This equation results from a first-order Taylor series expansion of Fick's Law with a temperature gradient. The first term in the right-hand side of the equation represents concentration-driven diffusion, the second term represents thermomigration, and the third is oxygen consumption due to chemical immobilization (i.e. absorption of oxygen to form chemical compounds). The oxygen diffusivity follows a standard Arrhenius law with temperature $T$, which itself depends on the spatial coordinate $y$ via the temperature gradient.

$$
D(T)=D_{0} \exp \left(\frac{-Q_{\mathrm{D}}}{k_{\mathrm{B}} T}\right)
$$

Suitable initial conditions to solve the differential equation system (5) by using finite differences can be defined at $t=0$ for stage I:

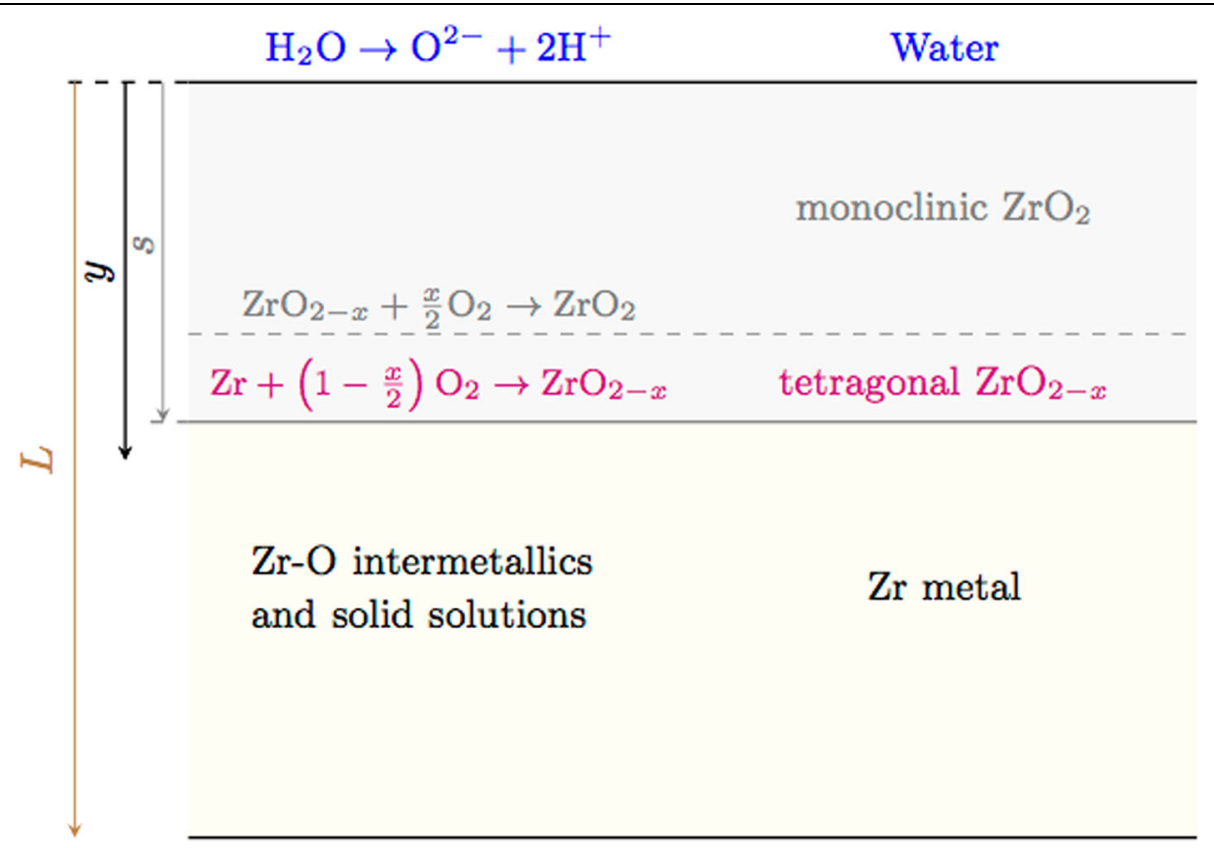

Fig. 3. Schematic representation of model parameters and evolved layers. The chemistry defining each layer is provided in simplified form in the left hand side, while the global layer designation is written in the right-hand side. 


$$
\text { I.C : }\left\{\begin{array}{l}
{[\mathrm{O}](y, 0)=0} \\
{[\mathrm{Zr}](y, 0)=[\mathrm{Zr}]_{0}}
\end{array}\right.
$$

where $[\mathrm{Zr}]_{0}$ is the (constant) initial zirconium concentration, obtained from its atomic density.

$$
\text { B.C : }\left\{\begin{array}{l}
{[O](0, t)=C_{0}} \\
\frac{\partial[0](L, t)}{\partial y}=0
\end{array}\right.
$$

where $C_{0}$ is the (constant) oxygen concentration, available from electrolysis on the water side, which is set by water chemistry, temperature, pressure, etc. The second equation reflects that no free oxygen can escape the clad into the fuel side. (i.e. $\left.J_{O}(L, t)=0\right)$. Our critical assumption for post-crack growth (stage II) regime is that the free oxygen from the environment side becomes immediately available on the oxide/metal interface via transport through the microcrack network and remains constant at $C_{0}$ as the crack grow. Therefore, the updated boundary condition becomes:

$$
[\mathrm{O}](s, t)=C_{0}
$$

A further simplification is to assume a single oxygen population that penetrates the clad by diffusion and is consumed due to the oxide reaction process. Oxygen diffusion results in a spatial depth distribution from which we obtain the concentration fraction $X$. Hence, we can obtain the spatial distribution of the emerging zirconium oxide phases based on the time evolution of $X$. The thickness of the oxide layer, $s$, is then selfconsistently obtained as the depth at which the oxygen in the clad is dissolved by the $\mathrm{Zr}$.

\section{Temperature Profile Update}

As shown in Eq. 5, $T(y)$ and $\mathrm{d} T / \mathrm{d} y$ are essential inputs with spatio-temporal distributions. As the oxide builds up, it reduces the heat transfer rate. Hence, heat flux evacuated radially from the fuel is:

$$
q=-\kappa(y) \frac{\mathrm{d} T}{\mathrm{~d} y} \leq \kappa_{\mathrm{Zr}} \frac{T_{\mathrm{f}}-T_{\mathrm{w}}}{L}
$$

Further, we assume a linear temperature profile through the clad with (at most) two separate phases (oxide plus metal):

$$
T(y)= \begin{cases}T_{\mathrm{w}}+\frac{T_{\mathrm{i}}-T_{\mathrm{w}}}{s} y & y<s \\ T_{\mathrm{i}}+\frac{T_{\mathrm{f}}-T_{\mathrm{i}}}{L-s} y & y \geq s\end{cases}
$$

where $T_{i}$ is the interface temperature obtained from Eq. 10 as:

$$
T_{\mathrm{i}}=\frac{\kappa_{\mathrm{r}}(L-s(t)) T_{\mathrm{w}}+s(t) T_{\mathrm{f}}}{\kappa_{\mathrm{r}}(L-s(t))+s(t)}
$$

where $\kappa_{r}=\kappa_{Z r O} O_{\kappa_{Z}}, L$ and $s(t)$ are the thermal conductivity ratio, the clad, and oxide layer thickness, and $T_{\mathrm{f}}$ and $T_{\mathrm{w}}$ are the temperatures on the fuel and water sides, respectively, which are taken to be constant. Hence, the temperature gradients at any given instant are obtained as:

$$
\left.\frac{\mathrm{d} T}{\mathrm{~d} y}\right|_{\mathrm{ox}}=\frac{T_{\mathrm{f}}-T_{\mathrm{w}}}{\kappa_{\mathrm{r}}(L-s(t))+s(t)}
$$

and

$$
\left.\frac{\mathrm{d} T}{\mathrm{~d} y}\right|_{\mathrm{Zr}}=\frac{T_{\mathrm{f}}-T_{\mathrm{w}}}{L-s(t)+\frac{s(t)}{\kappa_{\mathrm{r}}}}
$$

\section{Iterative Procedure}

The initial conditions are defined assuming a pure $\mathrm{Zr}$ layer, which sets the original temperature gradient and the rest of the associated temperaturedependent parameters. As the oxide layers grow, this assumption is updated accounting for the new three-layer metal/oxide system and temperature distributions. Hence, the simulation is carried out dynamically by iteration of time and space variations in diffusivity (D), thermal conductivity $(\kappa)$ and reaction rate $(k)$, as shown in Fig. 4.

The experimental kinetics of growth is shown to display a third power law relationship with the time. In this work, we first assume linear kinetics $(\beta=1)$. Subsequently, we iteratively vary $\beta$ until a $t^{1 / 3}$ dependence is recovered, adjusting the units of the rate constant $k$ in each case. The present model represents a simplified view of the oxidation process, which is nonetheless useful to gain qualitative insights into the kinetics of corrosion, and which will be augmented in future studies with a more fundamental description of the relevant processes.

\section{MODEL PARAMETERIZATION}

\section{Electronic Structure Calculations of Oxygen Diffusion in Zr Oxide}

By combining density functional theory (DFT) calculations and thermodynamic/kinetic continuum models, we have conducted a series of studies on the defect chemistry and transport properties of zirconia. The goal of these studies is to deduce physically

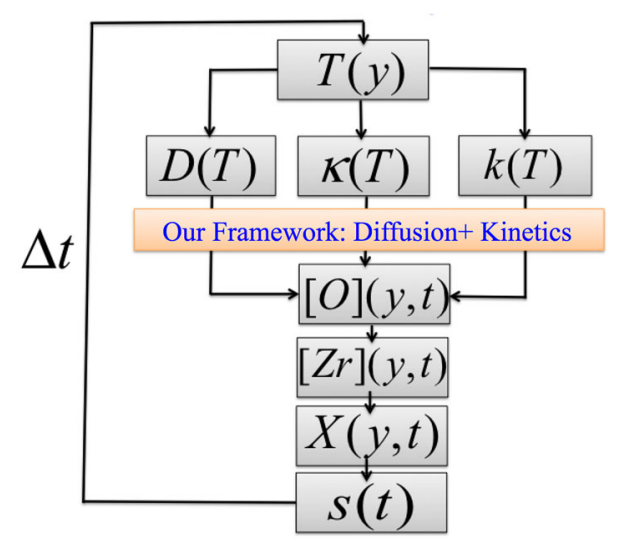

Fig. 4. Iterative procedure for the growth of oxide layer $s(t)$. 
based kinetic parameters, such as diffusion coefficients and surface reaction rates, and feed them to the higher-level kinetic models.

Conventional corrosion models are generally based on the assumption that doubly-charged oxygen vacancies and electrons are the two dominant types of intrinsic defects, ignoring the fact that the situation might change with varying temperature and oxygen chemical potential. In fact, the oxide layer is constantly exposed to a gradient in the chemical potential of oxygen from the oxygen-poor zone at the metal/oxide interface to the oxygen-rich zone at oxide/water interface. We assessed all possible intrinsic point defects in both tetragonal ${ }^{29}$ and monoclinic ${ }^{30}$ phases of $\mathrm{ZrO}_{2}$. Our results revealed that, for the tetragonal phase and under oxygen-poor conditions, the dominant defects are doubly-charged oxygen vacancies compensated by electrons. With continuous depletion of oxygen, the neutral oxygen vacancy takes over. For the monoclinic phase under oxygen-rich conditions and temperatures less than $500^{\circ} \mathrm{C}$, quadruple-charged zirconium vacancies prevail compensated by holes.

Oxygen diffusion inside the oxide film consists of various defect-mediated migration processes. Combining the formation free energies and migration barriers in a random-walk model, we predicted oxygen self-diffusion coefficients in tetragonal $\mathrm{ZrO}_{2}{ }^{31}$ Migration barriers of oxygen defects were calculated with the nudged elastic band (NEB) method. $^{32}$ The resulting oxygen self-diffusivity as a function of temperature and off-stoichiometry $(x$ in $\mathrm{ZrO}_{2-x}$ ) is reproduced from Ref. 31 in Fig. 5. At $x=10^{-4}$, the simulation results compare favorable to the experimental data. ${ }^{33}$ The origin of the deviation of the slope at this $x$ is the assumption of a pure $\mathrm{ZrO}_{2}$, which is not the case in the experiments. Accounting for impurities also results in better agreement in the slope, as discussed in Ref. 31.

Impurities in $\mathrm{ZrO}_{2}$ arise from the alloying elements that are present in the zirconium alloy. Aiming at quantifying the effect of metal dopants on oxidation and hydrogen pickup kinetics and identifying design principles for degradation-resistant zirconium alloys, we performed studies of common metal alloy elements dissolved in $\mathrm{ZrO}_{2}$. The existence of aliovalent metal dopants, even on the ppm level, could substantially change the defect chemistry and corrosion kinetics. In tetragonal $\mathrm{ZrO}_{2}$, we explicitly evaluated the effect of dissolved $\mathrm{Nb}^{34}$ DFT calculations show that $\mathrm{Nb}^{5+}$ substituting zirconium is the predominant $\mathrm{Nb}$ defect. Positively charged $\mathrm{Nb}$ dopants are compensated mainly by zirconium vacancies but to some extent also with electrons. This is also accompanied by a decrease in the concentration of oxygen vacancies. Kinetically, this is reflected in the enhancement of electron transport and the suppression of oxygen migration.
The values for oxygen diffusion ( $D_{0}$ and $\left.Q_{\mathrm{D}}\right)$ shown in Fig. 5 correspond to very small values of $x$. As a first-order approximation, we extrapolate the values of $D_{0}$ and $Q_{\mathrm{D}}$ to higher values of $x$ using the following expressions:

$$
\begin{aligned}
Q^{\mathrm{ox}}= & 1.182-0.152 \log (x)-0.036(\log (x))^{2}[\mathrm{eV}] \\
\log \left(D_{0}\right)= & -1.95+0.0304 \log (x)-0.1161(\log (x))^{2} \\
\times & {\left[\mathrm{D}^{\mathrm{ox}} \mathrm{in} \mathrm{cm}^{2} \mathrm{~s}^{-1}\right] }
\end{aligned}
$$

These expressions allow us to expand the nonstoichiometry range of oxygen diffusion in $\mathrm{ZrO}_{2-x}$ for $x$ values up to $\approx 0.02$.

\section{Mechanisms of Hydrogen Pickup and Diffusion on $\mathrm{Zr}$}

Hydrogen pickup and hydride formation are also important degradation processes in zirconium alloys that could potentially lead to embrittlement. Here, we focus on hydrogen pickup mechanisms, while hydride formation is addressed in more detail in "Summary" section. In order to understand how hydrogen is incorporated into the oxide passive film and how it enters the zirconium alloy, we have studied hydrogen-related defects in tetragonal (t-) $\mathrm{ZrO}_{2}{ }^{35}$ and monoclinic (m-) $\mathrm{ZrO}_{2} \cdot{ }^{30}$ Both in $\mathrm{t}-\mathrm{ZrO}_{2}$ and under oxygen-poor conditions, hydrogen is found to be more energetically favorable as a substitutional $\mathrm{H}^{+}$occupying an oxygen site. As

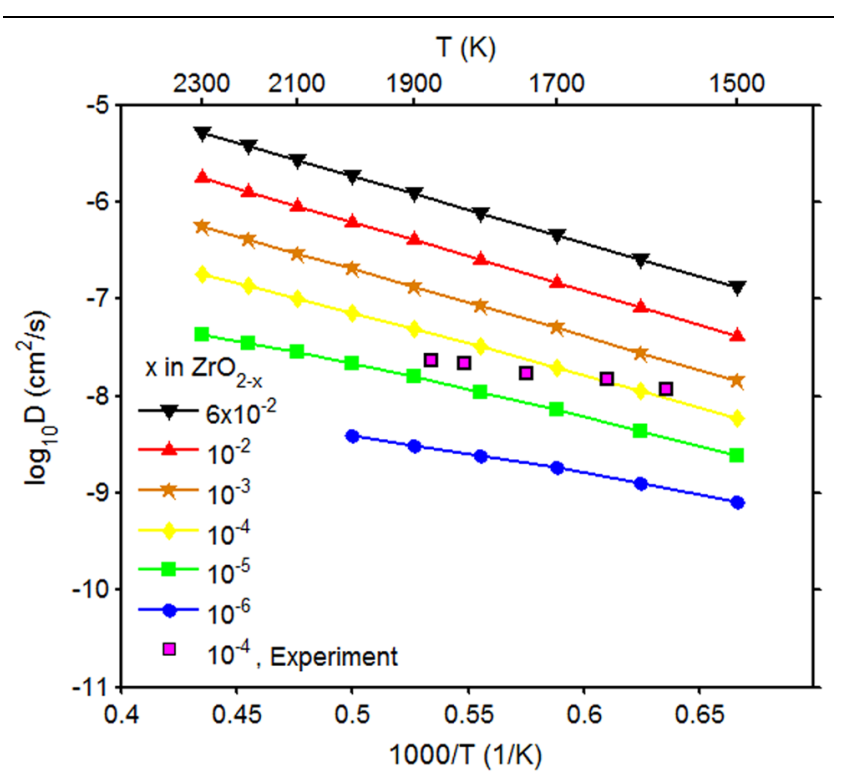

Fig. 5. Oxygen self-diffusion coefficient at constant off-stoichiometry, $x$, in tetragonal $\mathrm{ZrO}_{2-x}$ as a function of temperature. The experimental data are tracer diffusion coefficients from Ref. 33 scaled by a correlation factor of 0.65 . 
such, the concentration of substitutional hydrogen increases as one approaches the metal/oxide interface. Our results have also demonstrated that there is a tendency for hydrogen species to cluster at a zirconium vacancy site in oxygen-rich conditions, which could be potentially detrimental to the mechanical integrity of the oxide film, analogous to hydrogen embrittlement in the $\mathrm{Zr}$ metal.

By analyzing the effects of different alloying elements in $\mathrm{m}-\mathrm{ZrO}_{2}$, we have seen that the hydrogen solubility in the oxide exhibits a minimum with respect to the electron chemical potential, $\mu_{\mathrm{e}} \cdot{ }^{30} \mathrm{We}$ identified two doping strategies that could potentially suppress hydrogen uptake in the oxide. Dopants such as $\mathrm{Cr}$ could reduce the hydrogen solubility, while dopants such as $\mathrm{Nb}, \mathrm{Ta}, \mathrm{Mo}, \mathrm{W}$ or $\mathrm{P}$ could maximize $\mu_{e}$ and accelerate the $\mathrm{H}_{2}$ evolution kinetics at the surface. However, increasing $\mu_{\mathrm{e}}$ also results in enhanced hydrogen trapping at zirconium sites in $\mathrm{m}-\mathrm{ZrO}_{2}$, thus hindering its diffusion toward the oxide/metal interface. While $\mathrm{Nb}$ and $\mathrm{Cr}$ are already widely used in current zirconium alloys, Ta, Mo, $\mathrm{W}$ and $\mathrm{P}$ have been predicted in our work to accelerate the $\mathrm{H}_{2}$ evolution kinetics at the surface. So far, our studies have comprehensively covered defect chemistry related to oxidation kinetics and hydrogen incorporation in bulk $\mathrm{t}-\mathrm{ZrO}_{2}$ and $\mathrm{m}-\mathrm{ZrO}_{2}$. The computed formation and migration energies are sufficient for kinetic models where the passive film is treated as a homogeneous medium. However, extended defects, such as oxide/metal, oxide/water and oxide/secondary phase particles interfaces, also exist in the system and alter the energetics and kinetics nearby. Extending the current framework to account for structural variation and space-charge effects in the vicinity of these extended defects is the next step, and will provide more accurate quantifications of reaction rates and transport processes close to these critical interfaces.

\section{Simulation Parameters}

The values for simulation are listed in Table I.
The above parameters are taken from different sources including experiments dating back several decades, DFT calculations, etc., and have not been checked for self-consistency. For this reason, the results presented below are more a qualitative reflection of the oxidation process, rather than a rigorous quantitative analysis.

\section{RESULTS}

\section{Oxide Layer Growth Simulations}

Figure 6 shows the spatio-temporal evolution of the oxygen and atomic fraction according to the oxidation model. The thickness of the substoichiometric $\mathrm{ZrO}_{2-x}$ (tetragonal layer) is defined by the iso-contour $X=0.6644$. Figure 7 shows the evolution of the monoclinic oxide scale $\mathrm{s}(\mathrm{t})$ for $\beta=1$. The curve is compared with a $t^{1 / 3}$ power law, with very good agreement achieved. Further analysis shows that the closest agreement with a $t^{1 / 3}$ dependence is achieved for $\beta=0.7$. Figure 8 shows the oxygen concentration profiles during stage I before and after the oxide layer emerges.

According to experimental observations, stage II is seen to approximately begin at an oxide layer thickness of approximately $s \approx 80 \mu \mathrm{m}$. As mentioned earlier, this is brought about by fragmentation and cracking of the oxide scale, which occurs when pre-exisiting cracks propagate inward and reach the oxide/metal interface. At that point, oxygen and hydrogen ions from the water side become immediately available at the interface, so that diffusion through the oxide ceases to be the rate-limiting step for oxygen availability and oxide layer growth. During stage II, $s$ starts to scale quasilinearly with time, which is captured qualitatively by the model, as shown in the inset to Fig. 7 . It is worth noting that the model at the present stage captures the qualitative features of this breakaway growth regime, which is seen to strongly depend on coolant chemistry and irradiation conditions, among other factors.

Table I. Parameter values used in the kinetic model for oxide layer growth

\begin{tabular}{lcc}
\hline Parameter & Value & Units \\
\cline { 2 - 3 }$L$ & 600 & $\mu \mathrm{m}$ \\
$T_{\mathrm{f}}$ & 660 & $\mathrm{~K}$ \\
$T_{\mathrm{w}}$ & 600 & $\mathrm{~K}$ \\
$Q_{r x n}$ & $47.2^{36}$ & $\mathrm{kcal} \mathrm{mol}^{-1}$ \\
{$[\mathrm{O}]_{0}$} & $3.1 \times 10^{-36 \mathrm{a}}$ & $\mathrm{mol} \mathrm{l}^{-1}$ \\
{$[\mathrm{Zr}]_{0}$} & $7.17 \times 10^{-5} \mathrm{~b}$ & $\mathrm{Mol} \mathrm{l}^{-1}$ \\
$Q_{\mathrm{D}}$ & $4.45^{37}$ & $\mathrm{kcal} \mathrm{mol}^{-1}$ \\
$k_{0, r x n}$ & $6.1 \times 10^{438}$ & $\mathrm{kcal} \mathrm{mol}^{-1}$ \\
$\kappa_{\mathrm{ZrO}}$ & $2^{39} \mathrm{c}$ & $\mathrm{W} \mathrm{m}^{-1} \mathrm{~K}^{-1}$ \\
$\kappa_{\mathrm{Zr}}$ & $8.8+0.007 T+0.00003 T^{2}+0.3 T^{-140}$ & $\mathrm{~W} \mathrm{~m}^{-1} \mathrm{~K}^{-1}$ \\
\hline${ }^{\mathrm{a}}[\mathrm{O}]_{0}=0.05 \mathrm{ppb}=0.05 \mathrm{~g} / \mathrm{l}=3.1 \times 10^{-3} \mathrm{~mol} /{ }^{\mathrm{b}}{ }^{2}$ Assuming all $\mathrm{Zr}$ is available for oxidation reaction. ${ }^{\mathrm{c}}$ Almost insensitive to temperature. \\
\hline
\end{tabular}



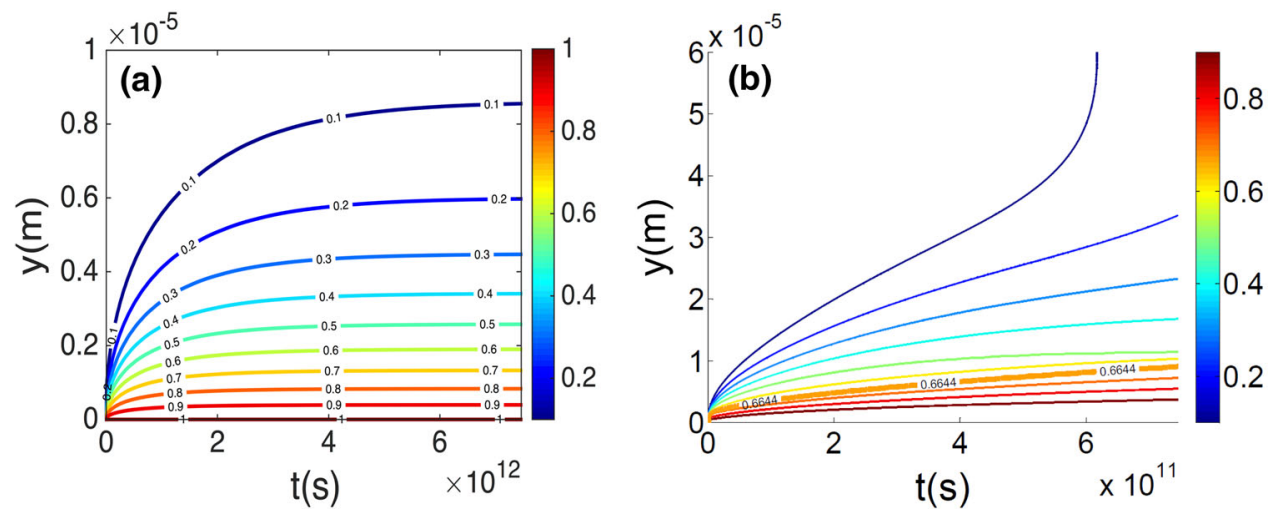

Fig. 6. (a) The time dependent evolution of oxygen concentration [O] and (b) non-stoichiometric ratio X (right). The tetragonal zirconia evolution is given by the 0.6644 iso-contour.

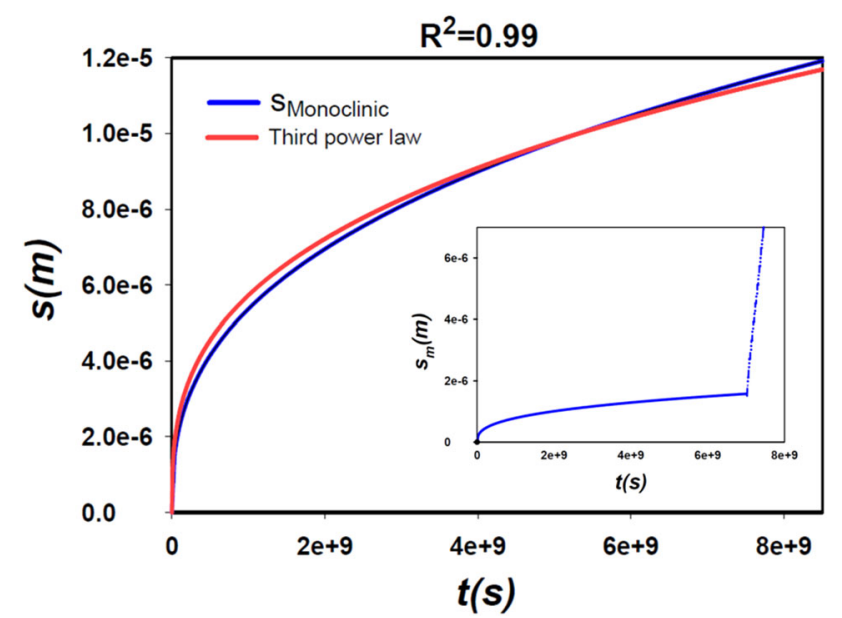

Fig. 7. The correlation of simulation growth with third power law. Inset Sudden jump in growth regime assuming $t_{\text {Crack }}=0.9 t_{\text {Simulation }}$.

\section{A Microscopic Model for the Hydrogen Kinetics in $\mathbf{Z r}$}

A critical aspect of the overall corrosion process is the coupling of hydrogen and oxygen transport through the oxide scale towards the metal substrate, where hydrogen further diffuses and forms hydride platelets. These platelets are preferentially oriented along the normal and tangential directions, and have a profound impact on the mechanical behavior and loss of ductility of the zircalloy. At this stage, these synergisms have not been fully established and hydrogen transport and precipitation are treated as independent processes. In this section, a rate theory model of hydride formation in $\mathrm{Zr}$ metal is proposed, without any coupling to the oxide scale kinetics for the moment.

Existing models ${ }^{41}$ for the hydrogen behavior (diffusion and hydride precipitation) in $\mathrm{Zr}$ use simplified expressions for reaction (precipitation and dissolution) rates to solve for the hydrogen concentration in the metal matrix (solid solution form) and the total concentration of hydrogen in all hydrides without regard to their sizes. While the partition of hydrogen into the matrix and the hydrides is important, the existing models do not provide more detailed information, such as the number density and the size distribution of the hydrides, which is valuable to mechanics modeling and failure probability analysis of the fuel-cladding system. Motivated by this, we have initiated an effort to develop a microscopic model for the hydrogen kinetics in Zr. Specifically, we use a cluster dynamics (CD) approach that establishes one equation for each size of the cluster (here hydride). The $\mathrm{CD}$ approach has been extensively used in modeling kinetic processes, e.g., defect clustering and helium bubble formation, in irradiated materials. ${ }^{42,43}$ With this approach, the hydrogen kinetics is described by the following system of equations:

$$
\left\{\begin{aligned}
\frac{\partial C_{1}(x, t)}{\partial t}= & \frac{\partial}{\partial x} J+2 \times k_{2}^{-} C_{2}+\sum_{n>2} k_{n}^{-} C_{n}-2 \times k_{1}^{+} C_{1}^{2} \\
& -\sum_{n>1} k_{n}^{+} C_{1} C_{n} \\
\left.\frac{\partial C_{n}(x, t)}{\partial t}\right|_{n>1}= & k_{n-1}^{+} C_{n} C_{n-1}+k_{n+1}^{-} C_{n+1}-k_{n}^{+} C_{1} C_{n}-k_{n}^{-} C_{n}
\end{aligned}\right\}
$$

where $C$ stands for the concentration $\left(\mathrm{nm}^{-3}\right), k_{n}^{+}$and $k_{n}^{-}$are the reaction constants for a cluster containing $n$-H atoms to capture and emit a H-atom, respectively. Here, we assume that the newly formed hydride has a fixed stoichiometry and hence the $\mathrm{H}$-number is sufficient to represent a hydride cluster and that a hydride cluster contains at least two H-atoms $\left(C_{1}\right.$ refers to the concentration of hydrogen in the metal matrix.) At present, our model uses the following expressions for the reaction rate constants: $k_{n}^{+}=4 \pi\left(r_{1}+r_{n}\right)\left(D_{1}+D_{n}\right)$ and $k_{n}^{-}=4 \pi\left(r_{1}+r_{n-1}\right)\left(D_{1}+D_{n-1}\right) C_{0} \exp \left(-\frac{E_{n}^{B}}{k_{B} T}\right)$, respectively, where $r$ is the radius, $D$ is the diffusivity $\left(D_{n}=D_{1}(\right.$ for $n=1)$ or $0($ for $\left.n>1)\right), \quad C_{0} \quad$ is the matrix atomic number density and $E_{n}^{B}$ is the binding energy of a $\mathrm{H}$-atom to the cluster. These expressions are most suitable for 3D diffusion-reaction systems 

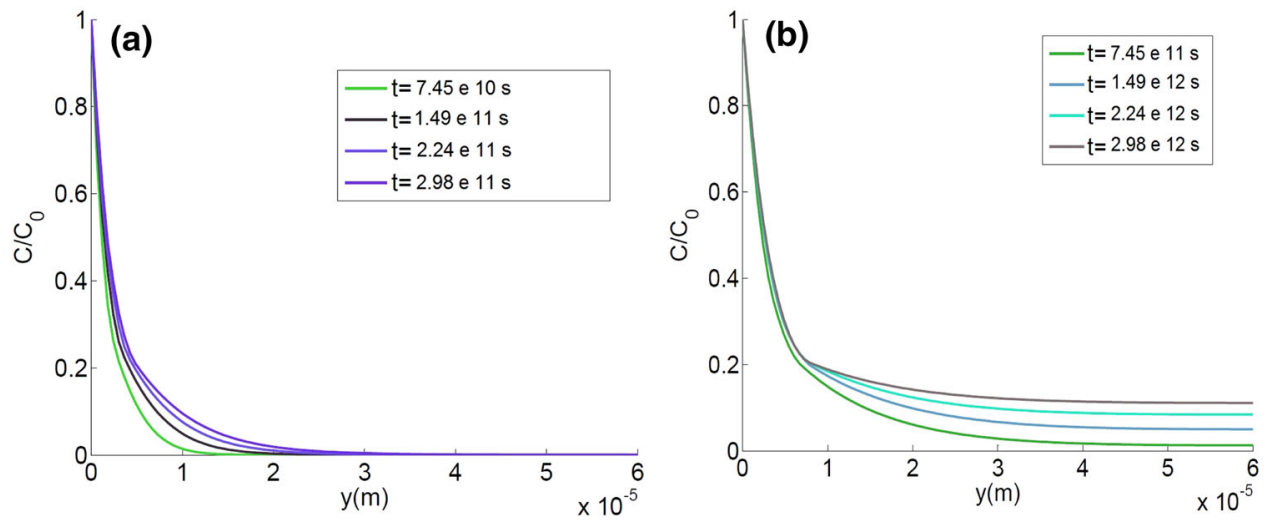

Fig. 8. (a) Spatial variation of oxygen concentration [O] in short- and (b) long-range time.

Table II. Parameters used in a first round simulation

$\mathrm{L}($ clad thickness) [nm]

$x$ (mesh grids) [nm]

$\nabla T$ (temperature gradient) $[\mathrm{K} / \mathrm{nm}]$

$T(x)$ (temperature at $x)[\mathrm{K}]$

$D_{0}$ (H diffusivity prefactor) $\left[\mathrm{nm}^{2} \mathrm{~s}^{-1}\right]$

$E_{\mathrm{m}}$ (migration energy of $\mathrm{H}$ ) $[\mathrm{eV}]$

$J_{\text {in }}$ (H pickup rate) $\left[\mathrm{nm}^{-2} \mathrm{~s}^{-1}\right]$

$r(\mathrm{n})$ (cluster radius) [nm]

$E_{n}^{B}$ (cluster binding energy) $[\mathrm{eV}]$

$Q$ (Soret coefficient) [K]

$$
\begin{gathered}
10^{6} \\
{\left[-10 .^{\wedge}([6:-.05: 54.9:-.1: 21.8:-.2: 0]) 0\right]} \\
-6 \times 10^{-5} \\
325+\nabla T \times x+273 \\
7 \times 10^{11} \\
0.47 \\
-0.02 \\
n^{1 / 3} \times 0.14 \\
0.435+(0.18-0.435)\left[n^{2 / 3}-(n-1)^{2 / 3}\right] /\left(2^{2 / 3}-1\right) \\
3000
\end{gathered}
$$

with near-spherical clusters, which may be a reasonable approximation to the early stage of hydride precipitation (or, very small precipitates). Alternative rate expressions will be used to account for the plate shape of large hydrides that has been reported in experiments. [e.g., Ref. 44]

Two unique features of the problem of the hydrogen kinetics in $\mathrm{Zr}$ cladding are the presence of a pronounced temperature gradient across the cladding thickness and an extra diffusion flux induced by the temperature gradient, the latter known as the Soret effect. The temperature gradient stipulates a spatial dependence of the hydrogen diffusivity and the reaction rate constants. The Soret effect modifies the overall hydrogen diffusion flux in the metal matrix as

$$
J=-D\left(\frac{\partial C}{\partial x}+Q \frac{C}{T^{2}} \frac{\mathrm{d} T}{\mathrm{~d} x}\right)
$$

where $Q$ is the Soret constant. After converting the partial differential equations into ordinary differential equations at different spatial positions and applying an all-zero initial condition, a constant (in-)flux, $J_{\text {in }}$, of hydrogen from the water side and a zero flux at the fuel-clad interface, we have performed a first round simulation of the hydrogen kinetics using the cluster dynamics model and the parameter values listed in Table I. Note that all the parameter values in Table II are known to be suitable for the hydride formation in $\alpha$ $\mathrm{Zr}$ according to, ${ }^{45}$ except those for $r(\mathrm{n})$ and $E_{n}^{B}$ which need to be finalized in the near future based on relevant experiments and/or atomistic studies.

Figure 9 shows the resulting dynamics of hydride cluster evolution as a function of depth for a $0.1-\mathrm{cm}-$ thick $\mathrm{Zr}$ metal clad in the presence of a temperature gradient and incorporating the Soret effect. At the beginning of the process, $\mathrm{H}$ atoms that flow into the material from the right (water side) boundary are transported rapidly to the left (fuel side) boundary. Subsequently, small hydride clusters/embryos containing a handful of $\mathrm{H}$-atoms start to form, first near the right boundary and then also towards the inner (left) positions, owing to monomer-capturing processes. The frame at $t=3,000,000 \mathrm{~s}$ ( $\sim 35$ days $)$ in Fig. 9 represents an even later stage where a pronounced $x$-dependence of the size and population of hydrides is clearly developed, with bigger and larger population of hydrides occurring near the right (water side) boundary. This preferential formation and growth of hydrides is qualitatively consistent with the general observations in experiments (e.g., Refs. 41 and 45).

We emphasize that the initial results presented here are intended to demonstrate the general behavior and the new functionalities of this model (e.g., providing separated information of size and number 

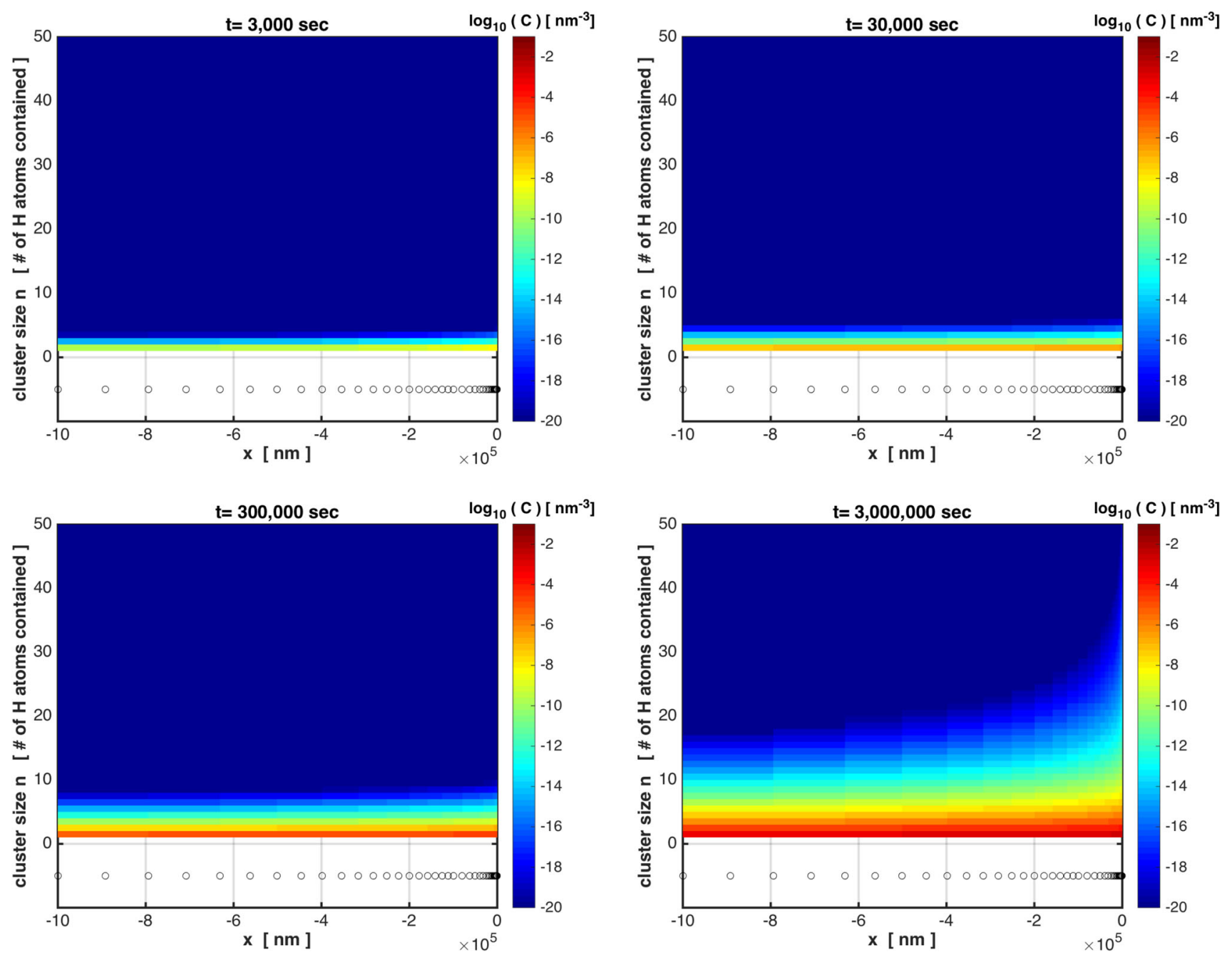

Fig. 9. Dynamic evolution of concentrations of dissolved $\mathrm{H}$ and hydride clusters (defined by number of $\mathrm{H}$ atoms contained) at different positions calculated with a temperature gradient and Soret effect. The spatial meshes are marked as circles. Note that the temperature linearly drops from $385^{\circ} \mathrm{C}$ on the left boundary to $325^{\circ} \mathrm{C}$ on the right boundary, and that there is a constant influx of $\mathrm{H}$ on the right boundary.

density of hydride clusters at different spatial positions within the cladding) that are not available from existing models, rather than to make detailed comparisons with any particular experiment. Full parameterization and thorough experimental validation of the model remain as a future set of goals.

\section{SUMMARY}

This work contains modeling results of zirconium oxide layer growth and the formation of hydride phases in $\mathrm{Zr}$ metal as a function of time. The models presented here represent fully integrated chemical reaction kinetics models, coupled to fundamental thermodynamic and kinetic data obtained by electronic structure calculations. The phase diagrams of the $\mathrm{Zr}$ oxide and hydride phases have been mapped out using cluster expansion Hamiltonians with structure identification, and provide the basis to formulate chemical models that account for stoichiometry and solubility behavior. Electronic structure methods also provide physical coefficients and parameters to fit the mesoscopic models.

In contrast with other more semi-empirical, qualitative models, the time dependence of the oxide layer thickness is not set a priori, but is an outcome of the calculations. We have found that the reaction order (which in general does not coincide with the stoichiometric coefficients of the reaction) sets the time exponent of the thickness growth dependence. Although a reaction order of unity comes close to furnishing the one-third power law dependence observed experimentally, we have found that a value of 0.7 provides the best fit. In terms of hydride formation, we have demonstrated a new cluster dynamics type of model that incorporates the hydrogen diffusion, clustering and thermal dissociation in the presence of a temperature gradient in a fundamentally self-consistent way, and provides 
additional information (e.g., size distribution and number density of hydride clusters) that are not available from existing models. We emphasize that there are still many open questions in regards to Zircalloy corrosion, such as irradiation effects, the impact of the alloy chemistry and microstructure, synergistic $\mathrm{H} / \mathrm{O}$ transport and chemistry, validation, etc. The models presented are an attempt to bring the full power of computational/physical modeling to a relatively old problem, using a new modeling philosophy supported by new numerical techniques and unprecedented computational power. The next phases of our modeling effort will involve bridging the oxide growth model with the hydride accumulation calculations.

\section{ACKNOWLEDGEMENTS}

This research was supported by the Consortium for Advanced Simulation of Light Water Reactors (CASL), an Energy Innovation Hub for Modeling and Simulation of Nuclear Reactors under US Department of Energy Contract No. DE-AC0500OR22725.

\section{REFERENCES}

1. B. Cox, Advances in Corrosion Science and Technology (Berlin: Springer, 1976), p. 173.

2. K. Forsberg, M. Limbäck, and A.R. Massih, Nucl. Eng. Des. 154, 157 (1995).

3. S. Kass, J. Nucl. Mater. 29, 315 (1969).

4. A.J.G. Maroto, R. Bordoni, M. Villegas, M.A. Blesa, A.M. Olmedo, A. Iglesias, and G. Rigotti, J. Nucl. Mater. 229, 79 (1996).

5. G.P. Sabol and S.B. Dalgaard, J. Electrochem. Soc. 122, 316 (1975).

6. T.R. Allen, R.J.M. Konings, and A.T. Motta, in Comprehensive Nuclear Materials, ed. J.M.K. Rudy (Oxford: Elsevier, 2012), pp. 49-68.

7. B.N.L. Srinivas, (Master of Science thesis, Department of Physics, Royal Institute of Technology, Stockholm, Sweden, 2012).

8. M. Kuroda, K. Yoshioka, S. Yamanaka, H. Anada, F. Nagase, and H. Uetsuka, J. Nucl. Sci. Technol. 37, 670 (2000).

9. K.E. Sickafus, H. Matzke, T. Hartmann, K. Yasuda, J.A. Valdez, P. Chodak III, M. Nastasi, and R.A. Verrall, J. Nucl. Mater. 274, 66 (1999).

10. R. Adamson, F. Garzarolli, B. Cox, A. Strasser, and P. Rudling, ZIRAT12 Special Topic Report (ANT International, Molnlycke, Sweden, 2007).

11. B. Cox, J. Nucl. Mater. 336, 331 (2005).

12. A. Varias and A. Massih, J. Nucl. Mater. 279, 273 (2000).

13. S.M. Allen and J.W. Cahn, Acta Metall. 27, 1085 (1979).

14. A. Van der Ven, J.C. Thomas, Q. Xu, and J. Bhattacharya, Math. Comput. Simul. 80, 1393 (2010).

15. A. Van De Walle and G. Ceder, Rev. Mod. Phys. 74, 11 (2002).
16. Q. Xu and A. Van der Ven, Intermetallics 17, 319 (2009).

17. A. Van der Ven, J.C. Thomas, Q. Xu, B. Swoboda, and D. Morgan, Phys. Rev. B 78, 104306 (2008).

18. J.M. Sanchez, F. Ducastelle, and D. Gratias, Phys. A 128, 334 (1984).

19. D. De Fontaine, Solid State Phys. 47, 33 (1994).

20. W. Zhong, D. Vanderbilt, and K. Rabe, Phys. Rev. Lett. 73, 1861 (1994)

21. W. Zhong, D. Vanderbilt, and K. Rabe, Phys. Rev. B 52, 6301 (1995).

22. J.C. Thomas and A. Van der Ven, Phys. Rev. B 88, 214111 (2013).

23. N. Ni, S. Lozano-Perez, J. Sykes, and C. Grovenor, Ultramicroscopy 111, 123 (2011).

24. Y. Dong, A.T. Motta, and E.A. Marquis, J. Nucl. Mater. 442,270 (2013).

25. B. Puchala and A. Van der Ven, Phys. Rev. B 88, 094108 (2013).

26. M.-H. Chen, B. Puchala, and A. Van der Ven, Calphad 51, 292 (2015).

27. J.C. Thomas and A. Van der Ven, Phys. Rev. B 90, 224105 (2014).

28. J. Abriata, J. Garces, and R. Versaci, Bull. Alloy Ph. Diagr. 7, 116 (1986)

29. M. Youssef and B. Yildiz, Phys. Rev. B 86, 144109 (2012).

30. M. Youssef, M. Yang, and B. Yildiz, Phys. Rev. Appl. 5, 014008 (2016).

31. M. Youssef and B. Yildiz, Phys. Rev. B 89, 024105 (2014).

32. G. Henkelman, B.P. Uberuaga, and H. Jónsson, J. Chem. Phys. 113, 9901 (2000).

33. K. Park and D. Olander, J. Electrochem. Soc. 138, 1154 (1991).

34. U. Otgonbaatar, W. Ma, M. Youssef, and B. Yildiz, J. Phys. Chem. C 118, 20122 (2014).

35. M. Youssef and B. Yildiz, Phys. Chem. Chem. Phys. 16, 1354 (2014).

36. J. Belle and M. Mallett, J. Electrochem. Soc. 101, 339 (1954).

37. M. Holz, S.R. Heil, and A. Sacco, Phys. Chem. Chem. Phys. $2,4740(2000)$.

38. J.P. Pemsler, J. Electrochem. Soc. 105, 315 (1958).

39. K. Schlichting, N. Padture, and P. Klemens, J. Mater. Sci. 36, 3003 (2001).

40. J. Fink and L. Leibowitz, J. Nucl. Mater. 226, 44 (1995).

41. O. Courty, A.T. Motta, and J.D. Hales, J. Nucl. Mater. 452, 311 (2014).

42. D. Xu, X. Hu, and B.D. Wirth, App. Phys. Lett 102, 011904 (2013).

43. D. Xu, B.D. Wirth, M. Li, and M.A. Kirk, Acta Mater. 60, 4286 (2012).

44. Y. Shinohara, H. Abe, T. Iwai, N. Sekimura, T. Kido, H. Yamamoto, and T. Taguchi, J. Nucl. Sci. Technol. 46, 564 (2009).

45. D.R. Olander, Fundamental Aspects of Nuclear Reactor Fuel Elements: Solutions to Problems, TID-26711-P2 (Springfield, Virginia: National Technical Information Service, Office of Public Affairs, Energy Research and Development Administration, U.S. Department of Commerce, 1976). http://www.osti.gov/scitech/servlets/purl/ 7290222 . 